\title{
Frozen-Thawed Embryo Transfer Success Rate is Affected by Age and Ovarian Response at Oocyte Aspiration Regardless of Blasto- mere Survival Rate
}

\author{
Yuval Bdolah ${ }^{1 *}$, Roni Zemet ${ }^{2 *}$, Einat Aizenman ${ }^{1}$, Francine Lossos ${ }^{1}$, Tali Bdolah Abram ${ }^{3}$, Yoel Shufaro ${ }^{4}$ \\ ${ }^{1}$ Reproductive Endocrinology Divisions and IVF Units, Department of Obstetrics \& Gynecology, Hadassah- \\ Hebrew University Medical Center, Mount Scopus and Ein Kerem, Jerusalem, Israel \\ ${ }^{2}$ Department of Obstetrics \& Gynecology, Chaim Sheba Medical Center, Tel Hashomer, Israel \\ ${ }^{3}$ The Hebrew University Medical School, Jerusalem, Israel \\ ${ }^{4}$ Infertility and IVF Unit, Beilinson's Hospital for Women, Rabin Medical Center, Petach Tikva 49100, Israel and \\ the Sackler Faculty of Medicine, Tel Aviv University, Tel Aviv, Israel
}

Presented orally at the Annual Meeting of the Israeli Fertility Association, Tel Aviv, Israel, May 20-21, 2013

*These authors have contributed equally to the preparation of this manuscript and should be considered joined first authors.

\begin{abstract}
Objective: To identify the factors influencing the success of frozen-thawed embryo transfers, whether originating directly from current cycles or from their matching fresh cycles.

Methods: Analysis of 273 frozen-thawed embryo transfer cycles and their matching fresh embryo transfer cycles, with respect to maternal, embryological and clinical factors, comparing successful to unsuccessful cycles.

Results: The cumulative clinical pregnancy and live birth rates following fresh ET and corresponding FETs were $50.5 \%$ and $38.8 \%$, respectively. No outcome measure differed between fresh and frozen ET's. Only maternal age, number of oocytes retrieved and fertilized, and number of cleaved embryos in the fresh cycle were correlated with a higher pregnancy or live birth rate in the FET cycle. None of the other parameters had any effect on the outcome. Pre-freezing embryo quality and blastomere survival rate had no effect on pregnancy/live birth rates.

Conclusion: Clinical pregnancy and live birth rates of fresh and frozen ETs were not significantly different. The only parameters that affected FET success were those resulting from the patient's age and ovarian reserve at the time of oocyte aspiration. Post-thawing blastomere survival rate and type of endometrial preparation for FET did not affect the success rate.
\end{abstract}

Keywords: Frozen thawed embryo transfer, Reproductive outcomes

\section{INTRODUCTION}

Reliable and successful embryo cryopreservation is a significant component of modern assisted reproduction technologies (ART). The first pregnancy resulting from the transfer of cleavage stage frozen-thawed human embryos was reported by Trounson \& Mohr (1983), and human blastocyst freezing was published in 1985 by Cohen et al. (Cohen et al., 1985). Embryo freezing facilitates the transfer of fewer embryos per cycle, thus minimizing the risk of multiple gestations, while maximizing the overall effectiveness of the ovarian stimulation cycle and oocyte pickup (OPU) procedure (Devroey et al., 2009; Pandian et al., 2009). In situations in which fresh embryo transfer (ET) is not advised or is contraindicated (for example unexpected illness or severe ovarian hyperstimulation syndrome), cryopreservation enables ET postponement. In addition, embryo cryopreservation offers fertility extension beyond the limitations of ovarian reserve or even lifespan (Shufaro \& Schenker, 2010) in certain cases. Consequently, at the end of the last decade, almost 1 out of 4 ART procedures performed in Europe was a frozen-thawed embryo transfer (FET) cycle (Ferraretti et al., 2012; Kupka et al., 2014).

It is possible to cryopreserve human embryos at the pronuclear stage, cleavage stage or at the early and expanded blastocyst stage (Michelmann \& Nayudu, 2006), either by slow-freezing or by vitrification. Although embryo vitrification is the most popular cryopreservation method, presently the greater mass of banked cryopreserved human embryos were frozen using slow-freezing protocols. These embryos are not always the best choice for ET and individual counseling should be offered regarding their specific reproductive potential.

Here we sought to determine and identify the factors that mostly affect the success rate of thawed embryos cryopreserved using slow freezing protocols. We looked into the parameters of each FET cycle, and its corresponding preceding fresh transfer cycle, comparing successful FETs to unsuccessful FETs, in order to define which factors are important to the reproductive potential of the frozen thawed embryos.

\section{MATERIALS AND METHODS \\ Study design and patients}

We performed a cohort study of data obtained from the Hadassah-Hebrew University Medical Center's Ein Kerem and Mount Scopus In Vitro Fertilization (IVF) units, Jerusalem, Israel. Patients who had a day 3 (slow freezing) FET between January 2007 and December 2010 were included. Cases of egg donation, preimplantation genetic diagnosis or fresh ET cancellation were excluded.

The parameters of each FET cycle were recorded as well as its preceding fresh day 3 ET cycle in which the excess embryos transferred in the FET cycle were cryopreserved. The fresh embryo transfer cycles were preformed from January 1999 through March 2010. For each patient only one OPU-fresh (day 3) ET cycle and the first subsequent (day 3) FET cycle were included in the study. The embryos were conceived by either intracytoplasmic sperm injection (ICSI) or conventional IVF.

Ovarian stimulation was induced by long down-regulation, short flare or antagonist protocols, with uFSH, rFSH, or hMG administered in accordance with conventional 
practice. When three or more follicles reached a diameter $\geq 17 \mathrm{~mm}$, oocyte maturation was induced by human chorionic gonadotrophin (5000 IU Pregnyl, Organon or 250 mcg Ovitrelle, Merck-Serono) administration. OPU occurred within 36 hours of hCG administration.

Embryo quality was evaluated 72 hours after insemination or ICSI based on the number of blastomeres and degree of fragmentation. Embryo morphology was scored according to Mandelbaum et al.: grade 1 - no fragments and equal blastomeres; grade 2 - equal blastomeres and $<20 \%$ fragments; grade 3 - no fragments and unequal blastomeres or $20-50 \%$ fragments, irrespective of blastomere equality; grade $4->50 \%$ fragments, irrespective of the equality of the blastomeres (Mandelbaum et al., 1987). Grade 4 embryos were not transferred.

In fresh ET cycles, progesterone luteal support was administered either intramuscularly (Gestone (Nordic Pharma) $50-100 \mathrm{mg} / \mathrm{d}$ ) or vaginally (Crinone (Merck-Serono) $1 / \mathrm{d}$; Endometrin (Ferring) $100 \mathrm{mg} \times 2 / \mathrm{d}$; or Utrogestean (Besin Laboratories) $200 \mathrm{mg} \mathrm{X3/d)} \mathrm{through} \mathrm{the} 10$ th week of gestation. Endometrial preparation for FET was achieved mainly by artificial preparation with exogenous estradiol (Estrofem (Novo Nordisk) 6-10 mg/d) and progesterone (intramuscular Gestone $50-100 \mathrm{mg} / \mathrm{d}$ or vaginal Crinone 1X2/d; Endometrin $200 \mathrm{mg} \mathrm{x2/d}$; or Utrogestean $400 \mathrm{mg}$ $\mathrm{X} 2 / \mathrm{d}$ ) or based on spontaneous ovulation. In FET cycles hormonal endometrial support (estrogen and progesterone) was continued through the $12^{\text {th }}$ week of gestation.

In the absence of any intervention or management outside the boundaries the standard practice and anonymous data collection, the study did not require an IRB approval.

\section{Embryo cryopreservation and thawing}

Supernumerary embryos not transferred fresh were cryopreserved by slow freezing protocol with 1, 2-propanediol (PROH) and sucrose (Lassalle et al., 1985). Embryos were first incubated in $1.5 \mathrm{M} \mathrm{PROH}$ freezing solution at room temperature for $10 \mathrm{~min}$, and then in $1.5 \mathrm{M} \mathrm{PROH}$ and $0.2 \mathrm{M}$ sucrose freezing solution at room temperature for another $10 \mathrm{~min}$. Cooling was carried out in a computerized machine at a rate of $-2^{\circ} \mathrm{C} / \mathrm{min}$ to $-8^{\circ} \mathrm{C}$, at which point seeding was induced manually. Cooling was continued at $-0.3^{\circ} \mathrm{C} / \mathrm{min}$ to $-30^{\circ} \mathrm{C}$, and then at $-50^{\circ} \mathrm{C} / \mathrm{min}$ to $-150^{\circ} \mathrm{C}$, before vial plunging into liquid nitrogen.

Thawing was performed by exposure to room temperature and immersion in water at $30^{\circ} \mathrm{C}$ for 30 seconds, followed by gradual cryoprotectant wash off at $37^{\circ} \mathrm{C}$. Embryo survival was defined if no more than $50 \%$ of the blastomeres suffered lysis after thawing.
Definition of outcomes and statistical analysis

Data on the medical and obstetrical history of the women and the outcome of the fresh transfer and FET cycles was collected from their medical records.

Implantation, clinical pregnancy, live birth, and miscarriage rates were calculated for the fresh embryo transfers and FET cycles. The implantation rate was calculated by dividing the number of visualized gestational sacs by the number of transferred embryos. Clinical pregnancy was defined if a fetal heart beat was observed.

We calculated that the enrollment of 212 patients would give the study a statistical power of $80 \%$ to detect an absolute difference of $12 \%$ in the rate of clinical pregnancies between fresh transfers and FETs, assuming an a of 0.05 with the use ofa two-tailed test.

For descriptive statistics purposes, we used the means \pm SD and range. In order to find the differences between fresh embryo transfer cycles and FET cycles, we used different statistical tools for quantitative and categorical variables. Quantitative variables were analyzed by the t-test and categorical variables were analyzed by the McNemar's test.

The assessment of parameters possibly related to the clinical pregnancy and live birth rates following FETs was accomplished by using logistic regression analysis. Odds ratios (ORs) with $95 \%$ confidence intervals (CIs) were presented. The logistic regression analysis of collected data was accomplished using chi-square test, Fisher's exact test and t-test.

All tests were two-sided, and a $P$ value of less than 0.05 was considered statistically significant.

Statistical analyses were performed using the SPSS software version 18.0 .

\section{RESULTS}

Data from 273 women, who had both a fresh and FET cycles from the same OPU during the study period, were included. Patient age at the time of fresh ET was $31.47 \pm$ 6.17 (range 18-44). ART etiologies included male factor $(62.3 \%)$, age-related infertility $(19 \%)$, unexplained infertility $(15.8 \%)$, tubal factor $(13.6 \%)$, and endometriosis $(4 \%)$. A total of $64.8 \%$ of patients achieved at least one pregnancy prior to the study period.

The ovarian stimulation protocols employed were: long agonist down-regulation (67.8\%), antagonist (19\%) and short agonist flare $(12.5 \%)$. The number of oocytes retrieved was $14.27 \pm 6$. ICSI and in vitro insemination were performed in 78.4 and 14.3 percent of cycles respectively. The number of fresh embryos transferred was $2.17 \pm 0.64$. A single embryo was transferred in $9.9 \%$, two embryos in

Table 1: Fresh and frozen-thawed embryo transfers $(n=273)$

\begin{tabular}{|l|c|c|c|}
\hline Parameter (mean \pm SD) & Fresh & Frozen & $P$ Value \\
\hline Age at ET (range) & $31.47 \pm 6.17(18-44)$ & $32.71 \pm 6.09(19-45)$ & $P<0.0001 *$ \\
\hline Number of transferred embryos & $2.17 \pm 0.64$ & $2.08 \pm 0.75$ & $P=0.096 *$ \\
\hline Embryo quality (range) & $1.53 \pm 0.41(1-2.75)$ & $1.86 \pm 0.45(1-2.83)$ & $P<0.0001 *$ \\
\hline Endometrial thickness (cm) & $1.1 \pm 0.23$ & $0.99 \pm 0.2$ & $P<0.0001 *$ \\
\hline Peak blood estradiol level (pMol/L) & $5703.54 \pm 3264$ & $1510.57 \pm 1510$ & $P<0.0001 *$ \\
\hline Positive pregnancy test (\%) & $110(40.3 \%)$ & $97(35.5 \%)$ & $0.271 * *$ \\
\hline Clinical pregnancy (\%) & $89(32.6 \%)$ & $76(27.8 \%)$ & $0.255^{* *}$ \\
\hline Miscarriages (\%) & $29(26.4 \%)$ & $27(27.8 \%)$ & $0.983 * *$ \\
\hline Deliveries (\% out of total cycles) & $67(24.5 \%)$ & $54(19.8 \%)$ & $0.208 * *$ \\
\hline Implantation rate (\%) & $19.17 \%$ & $17.49 \%$ & $0.511 *$ \\
\hline$*$ Paired t test **McNemar test. & & & \\
\hline
\end{tabular}


Table 2: Maternal variables and deliveries in FET cycles $(n=273)$

\begin{tabular}{|c|c|c|c|}
\hline \multirow{3}{*}{ Parameter } & \multicolumn{2}{|c|}{ Live birth } & \multirow{3}{*}{$P$ value } \\
\hline & Yes & No & \\
\hline & \multicolumn{2}{|c|}{ n (\%) } & \\
\hline $\begin{array}{l}\text { Age at OPU (n) } \\
\text { Age }<30(108) \\
30 \leq A g e \leq 35 \quad(93) \\
36 \leq A g e \leq 40 \quad(47) \\
\text { Age } \geq 41(25)\end{array}$ & $\begin{array}{c}30(55.6 \%) \\
13(24.1 \%) \\
9(16.7 \%) \\
2(3.7 \%) \\
\end{array}$ & $\begin{array}{l}78(35.6 \%) \\
80(36.5 \%) \\
38(17.4 \%) \\
23(10.5 \%) \\
\end{array}$ & $0.042 * *$ \\
\hline $\begin{array}{l}\text { Age at FET (n) } \\
\text { Age }<30(89) \\
30 \leq \text { Age } \leq 35(92) \\
36 \leq \text { Age } \leq 40(56) \\
\text { Age } \geq 41(36)\end{array}$ & $\begin{array}{c}24(44.4 \%) \\
16(29.6 \%) \\
12(22.2 \%) \\
2(3.7 \%) \\
\end{array}$ & $\begin{array}{l}65(29.7 \%) \\
76(34.7 \%) \\
44(20.1 \%) \\
34(15.5 \%)\end{array}$ & $0.048 * *$ \\
\hline $\begin{array}{l}\text { Previous pregnancy (\%) } \\
\text { Previous live birth }(\%)\end{array}$ & $\begin{array}{l}31.5 \\
25.9 \\
\end{array}$ & $\begin{array}{l}36.1 \\
18.3 \\
\end{array}$ & $\begin{array}{l}0.527 * \\
0.206 * \\
\end{array}$ \\
\hline $\begin{array}{l}\text { Infertility etiology } \\
\text { Male } \\
\text { Tubal } \\
\text { Endometriosis } \\
\text { Advanced age } \\
\text { Unexplained }\end{array}$ & $\begin{array}{c}37(68.5 \%) \\
4(7.4 \%) \\
1(1.9 \%) \\
7(13.0 \%) \\
9(16.7 \%) \\
\end{array}$ & $\begin{array}{c}133(61.0 \%) \\
33(15.1 \%) \\
10(4.6 \%) \\
45(20.5 \%) \\
34(15.6 \%) \\
\end{array}$ & $\begin{array}{c}0.308^{*} \\
0.138^{*} \\
0.699^{*} * \\
0.204^{*} \\
0.847^{*}\end{array}$ \\
\hline Pregnancy In corresponding Fresh ET & 35.2 & 32 & $0.651 *$ \\
\hline
\end{tabular}

$66.3 \%$, and 3 embryos in $21.2 \%$ of the cycles. Surplus embryos were frozen on day 3 . The number of embryos frozen was $5.68 \pm 3.94$ (range 1-22).

In $91.2 \%$ of frozen-thawed cycles, the endometrium was prepared with estradiol (oral, vaginal, trans-dermal or a combination of the above) while in $7.7 \%$ natural cycle follow-up with or without hCG induction of ovulation was performed. The mean number of frozen-thawed embryos transferred was $2.08 \pm 0.75$. A single embryo was transferred in $19.4 \%$, two in $57.1 \%$, and 3 in $19.4 \%$ of cycles. The cumulative clinical pregnancy and live birth rates, achieved by the combination of a fresh cycle, followed by a frozen-thawed cycle were $50.5 \%$ and $38.8 \%$, accordingly.

When comparing the overall clinical pregnancy, abortion and live birth rates between fresh and frozen-thawed cycles, no significant difference was seen (table 1). That is despite a disadvantage in the mean quality of embryos (prior to freezing), and an insignificant disadvantage in the number of embryos transferred in the frozen-thawed group.

We further analyzed the maternal, embryonic and treatment variables that affected the clinical pregnancy and live birth rates in the frozen-thawed cycles (tables 2-4). Maternal age only was correlated with a higher live birth (but not clinical pregnancy) rate in the FET cycles, while neither severity or parity nor pregnancy in the fresh cycle were correlated with the outcome of the frozen-thawed cycles (table 2). Similarly, the etiology of infertility had no effect on success rates. Embryo quality (prior to freezing) and blastomere survival rate after thawing were unrelated to clinical pregnancy or live birth after FET (table 3).

Analyzing the treatment parameters that affected success rates with frozen-thawed embryos revealed that only a few parameters were significant (table 4). The number of oocytes retrieved and fertilized, the number of cleaved embryos, and the number of embryos appropriate for freezing correlated with a higher clinical pregnancy rate in the FET cycles, while only the last variable correlated with a higher live birth rate. In logistic regression analyses only the number of embryos available for cryopreservation was significantly associated with clinical pregnancy and live birth rates in the FET cycle. All these parameters are consequential to patient age and ovarian reserve. Neither type of IVF stimulation protocol used in the fresh cycle, number of thawed embryos transferred, endometrial preparation protocol, endometrial thickness, nor estradiol and progesterone route of administration, had any effect on success rates.

\section{DISCUSSION}

In the present study, we analyzed the outcome measures of FET cycles and the corresponding fresh cycles in the same patients in order to elucidate the factors contributing to FET success. Our findings indicate that implantation, miscarriage, clinical pregnancy, and live birth rates following FETs and fresh embryo transfers were similar. Cryopreserved embryos were not inferior to the fresh embryos from the same oocyte cohort, despite a significant disadvantage

Table 3: Embryonic parameters in FET cycles, clinical pregnancies and deliveries $(\mathbf{n}=\mathbf{2 7 3})$

\begin{tabular}{|l|c|c|c|c|c|c|}
\hline \multirow{2}{*}{ Parameter } & \multicolumn{2}{|c|}{ Clinical pregnancy } & \multirow{2}{|c|}{ Live Birth } & \multicolumn{2}{|c|}{$P$ value } \\
\cline { 2 - 6 } & Yes & No & & \multicolumn{2}{|c|}{ Yes } & \\
\hline Embryo quality before freezing & $1.78 \pm 0.43$ & $1.89 \pm 0.46$ & $0.066 *$ & $1.82 \pm 0.39$ & $1.87 \pm 0.47$ & $0.274^{*}$ \\
\hline $\begin{array}{l}\text { Blastomere survival rate after } \\
\text { thawing(\%) }\end{array}$ & $77.42 \%$ & $76.85 \%$ & $0.845^{*}$ & $78.32 \%$ & $76.70 \%$ & $0.617^{*}$ \\
\hline *t-test & & & & & \\
\hline
\end{tabular}




\begin{tabular}{|c|c|c|c|c|c|c|}
\hline \multirow[t]{2}{*}{ Parameter } & \multicolumn{2}{|c|}{ Clinical Pregnancy } & \multirow[t]{2}{*}{$P$ value } & \multicolumn{2}{|c|}{ Live Birth } & \multirow[t]{2}{*}{$P$ value } \\
\hline & Yes & No & & Yes & No & \\
\hline Fresh cycle protocol type & & & $0.34 *$ & $0.21 *$ & & \\
\hline Oocytes retrieved & $15.46 \pm 6.4$ & $13.81 \pm 5.8$ & $0.04 * *$ & $15.31 \pm 6.4$ & $14.01 \pm 5.9$ & $0.16 * *$ \\
\hline Oocytes fertilized & $11.33 \pm 5.4$ & $9.65 \pm 4.7$ & $0.01 * *$ & $11.09 \pm 5.3$ & $9.88 \pm 4.8$ & $0.10 * *$ \\
\hline Cleaved embryos & $9.45 \pm 4.3$ & $8.34 \pm 3.8$ & $0.04 * *$ & $9.41 \pm 4.4$ & $8.46 \pm 3.8$ & $0.12 * *$ \\
\hline $\begin{array}{l}\text { Number of embryos frozen in fresh } \\
\text { cycle }\end{array}$ & $6.64 \pm 4.4$ & $5.31 \pm 3.7$ & $0.01 * *$ & $6.7 \pm 4.4$ & $5.43 \pm 3.8$ & $0.03 * *$ \\
\hline Number of embryos thawed & $2.67 \pm 1.1$ & $2.98 \pm 1.5$ & $0.11 * *$ & $2.70 \pm 1.1$ & $2.94 \pm 1.5$ & $0.27 * *$ \\
\hline $\begin{array}{l}\text { Number of embryos } \\
\text { transferred after thawing }\end{array}$ & $2.01 \pm 0.7$ & $2.11 \pm 0.7$ & $0.33 * *$ & $2.00 \pm 0.6$ & $2.11 \pm 0.8$ & $0.36 * *$ \\
\hline Assisted hatching performed (\%) & 6.6 & 14.7 & $0.07 *$ & 7.4 & 13.7 & $0.21 *$ \\
\hline \multicolumn{7}{|l|}{$\begin{array}{l}\text { Mode of endometrial preparation } \\
\text { for FET }\end{array}$} \\
\hline Spontaneous $(n=21)$ & $6(7.9 \%)$ & $15(7.7 \%)$ & $1.0 * * *$ & $5(9.3 \%)$ & $16(7.4 \%)$ & $0.67 * * *$ \\
\hline Artificial $(n=249)$ & $70(92.1 \%)$ & $179(91.8 \%)$ & & $49(90.7 \%)$ & $200(92.2 \%)$ & \\
\hline Mean endometrial thickness $(\mathrm{cm})$ & 0.972 & 0.9914 & $0.47 * *$ & 0.9881 & 0.9854 & $0.93 * *$ \\
\hline Max Estradiol (pmol/l) & 1724.7 & 1427 & $0.15^{* *}$ & 1720.6 & 1458.1 & $0.26 * *$ \\
\hline$* * *$ Fisher's e & act test & & & & & \\
\hline
\end{tabular}

in the mean morphological grade of embryos transferred following cryopreservation and an insignificantly higher number of embryos transferred in the fresh cycle (table 1).

When embryos are cryopreserved, their chance of implantation may be adversely affected by the freeze and thawing procedure (Edgar et al., 2000). Historically, cryopreserved embryos were considered the result of de-selection, after the best quality embryos are transferred fresh and the "second best" ones are cryopreserved (Check et al., 2001; Maheshwari et al., 2012). However, in more recent studies, cryopreserved embryos were not inferior to fresh ones (Feng et al., 2012; Veleva et al., 2013).

The similar pregnancy and live birth rates following frozen and fresh embryo transfers, can also be explained by the morphologic, genetic and biochemical adverse effects on the endometrium caused by the supra-physiologic estradiol levels during ovarian stimulation causes, leading to lower implantation rates (Shapiro et al., 2011). In contrast, the uterine and maternal endocrine environment into which cryopreserved embryos are transferred is more likely to mimic the natural cycle (Devroey et al., 2004; Haouzi et al., 2009; Paulson, 2011; Santos et al., 2010). In our study, the mean peak estradiol level in the fresh cycles was significantly higher than in the FET cycles. In addition, the freezing and thawing process may select embryos with a higher implantation potential (Shih et al., 2008).

The average age of patients participating in our study was relatively young (average 31.47 ) contributing to a high number of good quality embryos available for fresh as well as frozen ETs.

The analysis of our data revealed that the clinical pregnancy rate after FET was primarily dependent on the parameters of the cycles in which the oocytes were aspirated; the number of oocytes retrieved and fertilized, and the numbers of cleaved embryos available for transfer and cryopreservation. The live birth rate after FETs was found to be associated with patient age at time of both OPU and FET, and the number of viable frozen embryos (table 2). All these parameters are a direct function of patient age, ovarian reserve and intrinsic properties of the oocyte. These findings are not surprising and correlate with pre- vious published studies (Toner et al., 1991; Wang et al., 2001). Morphological embryo grading prior to freezing was associated with the clinical pregnancy rate, but not reaching significance (table 3$)(P=0.066)$. This is also consistent with previous publications (Mandelbaum et al., 1987; Salumets et al., 2006; Schalkoff et al., 1993). The type of endometrial preparation had no impact on the chance to conceive (table 4), in agreement with the existing literature (Ghobara \& Vandekerckhove, 2008).

Contrary to other studies published (Edgar et al., 2000; El-Toukhy et al., 2003; Vahratian et al., 2003; Wang et al., 2001), we found that the survival rate of blastomeres after thawing, the number of thawed embryos transferred and the outcome of prior fresh embryo transfer did not differ between patients who conceived and delivered, and those who did not. These novel observations were validated in our study by comparing cycles in the same patient thus eliminating individual differences between patients.

We believe that our results, the clinical and embryological parameters that were found to be associated with pregnancy and live birth in FET cycles, and those that were not, converge into one single explanation. Our hypothesis is that these results are explained by the theory that every embryo has its own independent implantation potential, which is a result of its intrinsic properties. Thus, implantation in FET cycles is mainly associated with parameters of the corresponding OPU cycle related to patient age, ovarian reserve and oocyte quality; the number of oocytes retrieved and fertilized, and the number of cleaved embryos available for transfer and cryopreservation. Pre-freezing embryo morphological grading was found not to be of value, resembling the limitations of this tool in predicting implantation in fresh ETs (Milki et al., 2002; Neuber et al., 2006; Seli et al., 2010; Vergouw et al., 2008). Since most patients have a normal endometrium, preparation based on spontaneous ovulation or artificial preparation with only a mild elevation of peak blood estradiol produces the same results. The occurrence of pregnancy in the fresh ET cycle or at any other time was found to have no relevance to the chance of each frozen thawed embryo to implant, which is totally independent from the rest of the cohort. The same 
applies to the number of embryos actually transferred after thawing.

Finally, the most interesting finding is that the blastomere survival rate was identical in transfers resulting in pregnancy and live birth and in unsuccessful transfers (table 3). If an embryo survived thawing and was defined suitable for ET (i.e. cell lysis $\leq 50 \%$ ), its implantation chance was unaffected by the blastomere survival rate, contrary to the published literature (Edgar et al., 2000; El-Toukhy et al., 2003). This discrepancy is probably due to the fact that the embryos included in our study were frozen and thawed after embryo slow freezing protocols were already optimized and reached their peak efficiency. Assuming that euploid embryos with a good implantation potential do contain aneuploid blastomeres, it is possible that the slow freezing and thawing may damage mainly these blastomeres without adversely affecting their independent implantation potential.

\section{CONCLUSIONS}

Our results indicate that the outcome of frozen embryo transfers cycles were good and comparable with fresh cycles even in the case of slow freezing. The parameters mainly associated with pregnancy and live birth after FETs were patient age and ovarian reserve at the time of OPU, as reflected by the number of oocytes retrieved and fertilized, and the number of cleaved embryos available for transfer. Pre-freezing embryo grading was of borderline significance. Contrary to published data, the number of transferred thawed embryos and the blastomere survival rate had no impact on the chance to conceive and deliver.

\section{CONFLICT OF INTERESTS}

No conflict of interest have been declared.

\section{Corresponding author:}

Yoel Shufaro

Infertility and IVF Unit, Beilinson's Hospital for Women, Rabin Medical Center, Petach Tikva, Israel

The Sackler Faculty of Medicine, Tel Aviv University

Tel Aviv - Israel

E-mail: yoelsh1@clalit.org.il

\section{REFERENCES}

Check JH, Choe JK, Nazari A, Fox F, Swenson K. Fresh embryo transfer is more effective than frozen for donor oocyte recipients but not for donors. Hum Reprod. 2001;16:1403-8.

Cohen J, Simons RF, Fehilly CB, Fishel SB, Edwards RG, Hewitt J, Rowlant GF, Steptoe PC, Webster JM. Birth after replacement of hatching blastocyst cryopreserved at expanded blastocyst stage. Lancet. 1985;325:647.

Devroey P, Bourgain C, Macklon NS, Fauser BC. Reproductive biology and IVF: ovarian stimulation and endometrial receptivity. Trends Endocrinol Metab. 2004;15:84-90.

Devroey P, Fauser BC, Diedrich K, Evian Annual Reproduction (EVAR) Workshop Group 2008. Approaches to improve the diagnosis and management of infertility. Hum Reprod Update. 2009;15:391-408.

Edgar DH, Bourne H, Speirs AL, McBain JC. A quantitative analysis of the impact of cryopreservation on the implantation potential of human early cleavage stage embryos. Hum Reprod. 2000;15:175-9.

El-Toukhy T, Khalaf Y, Al-Darazi K, Andritsos V, Taylor A, Braude P. Effect of blastomere loss on the outcome of frozen embryo replacement cycles. Fertil Steril. 2003;79:1106-11.
Feng G, Zhang B, Zhou H, Shu J, Gan X, Wu F, Deng X. Comparable clinical outcomes and live births after single vitrified-warmed and fresh blastocyst transfer. Reprod Biomed Online. 2012; 25:466-73.

Ferraretti AP, Goossens V, de Mouzon J, Bhattacharya S, Castilla JA, Korsak V, Kupka M, Nygren KG, Nyboe Andersen A, European IVF-monitoring (EIM); Consortium for European Society of Human Reproduction and Embryology (ESHRE). Assisted reproductive technology in Europe, 2008: results generated from European registers by ESHRE. Hum Reprod. 2012;27:2571-84.

Ghobara T, Vandekerckhove P. Cycle regimens for frozen-thawed embryo transfer. Cochrane Database Syst Rev. 2008, 1:CD003414.

Haouzi D, Assou S, Mahmoud K, Tondeur S, Reme T, Hedon B, De Vos J, Hamamah S. Gene expression profile of human endometrial receptivity: comparison between natural and stimulated cycles for the same patients. Hum Reprod. 2009;24:1436-45.

Kupka MS, Ferraretti AP, de Mouzon J, Erb K, D'Hooghe T, Castilla JA, Calhaz-Jorge C, De Geyter C, Goossens V, European IVF-Monitoring Consortium, for the European Society of Human Reproduction and Embryology. Assisted reproductive technology in Europe, 2010: results generated from European registers by ESHRE. Hum Reprod. 2014;29:2099-113.

Lassalle B, Testart J, Renard JP. Human embryo features that influence the success of cryopreservation with the use of 1,2 propanediol. Fertil Steril. 1985;44:645-51.

Maheshwari A, Pandey S, Shetty A, Hamilton M, Bhattacharya S. Obstetric and perinatal outcomes in singleton pregnancies resulting from the transfer of frozen thawed versus fresh embryos generated through in vitro fertilization treatment: a systematic review and meta-analysis. Fertil Steril. 2012;98:368-77 e1-9.

Mandelbaum J, Junca AM, Plachot M, Alnot MO, Alvarez S, Debache C, Salat-Baroux J, Cohen J. Human embryo cryopreservation, extrinsic and intrinsic parameters of success. Hum Reprod. 1987; 2:709-15.

Michelmann HW, Nayudu P. Cryopreservation of human embryos. Cell Tissue Bank. 2006; 7:135-41.

Milki AA, Hinckley MD, Gebhardt J, Dasig D, Westphal LM, Behr B. Accuracy of day 3 criteria for selecting the best embryos. Fertil Steril. 2002;77:1191-5.

Neuber E, Mahutte NG, Arici A, Sakkas D. Sequential embryo assessment outperforms investigator-driven morphological assessment at selecting a good quality blastocyst. Fertil Steril. 2006; 85:794-6.

Pandian Z, Bhattacharya S, Ozturk O, Serour G, Templeton $A$. Number of embryos for transfer following in-vitro fertilisation or intra-cytoplasmic sperm injection. Cochrane Database Syst Rev. 2009;2:CD003416.

Paulson RJ. Hormonal induction of endometrial receptivity. Fertil Steril. 2011;96:530-5.

Salumets A, Suikkari AM, Makinen $S$, Karro $H$, Roos A, Tuuri T. Frozen embryo transfers: implications of clinical and embryological factors on the 
pregnancy outcome. Hum Reprod. 2006; 21:2368 -74 .

Santos MA, Kuijk EW, Macklon NS. The impact of ovarian stimulation for IVF on the developing embryo. Reproduction. 2010;139:23-34.

Schalkoff ME, Oskowitz SP, Powers RD. A multifactorial analysis of the pregnancy outcome in a successful embryo cryopreservation program. Fertil Steril. 1993;59:1070-4.

Seli E, Robert C, Sirard MA. OMICS in assisted reproduction: possibilities and pitfalls. Mol Hum Reprod. 2010;16:51330.

Shapiro BS, Daneshmand ST, Garner FC, Aguirre M, Hudson $C$, Thomas S. Evidence of impaired endometrial receptivity after ovarian stimulation for in vitro fertilization: a prospective randomized trial comparing fresh and frozen-thawed embryo transfer in normal responders. Fertil Steril. 2011;96(2):344-8.

Shih W, Rushford DD, Bourne H, Garrett C, McBain JC, Healy DL, Baker HW. Factors affecting low birthweight after assisted reproduction technology: difference between transfer of fresh and cryopreserved embryos suggests an adverse effect of oocyte collection. Hum Reprod. 2008; 23:1644-53.

Shufaro Y, Schenker JG. Cryopreservation of human ge- netic material. Ann N Y Acad Sci. 2010; 1205:220-4. Toner JP, Brzyski RG, Oehninger S, Veeck LL, Simonetti S, Muasher SJ. Combined impact of the number of pre-ovulatory oocytes and cryopreservation on IVF outcome. Hum Reprod. 1991;6:284-9.

Trounson A, Mohr L. Human pregnancy following cryopreservation, thawing and transfer of an eight-cell embryo. Nature. 1983;305:707-9.

Vahratian A, Schieve LA, Reynolds MA, Jeng G. Live-birth rates and multiple-birth risk of assisted reproductive technology pregnancies conceived using thawed embryos, USA 1999-2000. Hum Reprod. 2003;18:1442-8.

Veleva Z, Orava M, Nuojua-Huttunen S, Tapanainen JS, Martikainen $\mathrm{H}$. Factors affecting the outcome of frozen-thawed embryo transfer. Hum Reprod. 2013;28:2425-31.

Vergouw CG, Botros LL, Roos P, Lens JW, Schats R, Hompes PG, Burns DH, Lambalk CB. Metabolomic profiling by near-infrared spectroscopy as a tool to assess embryo viability: a novel, non-invasive method for embryo selection. Hum Reprod. 2008;23:1499 -504 .

Wang JX, Yap YY, Matthews CD. Frozen-thawed embryo transfer: influence of clinical factors on implantation rate and risk of multiple conception. Hum Reprod. 2001;16:2316-9. 